\title{
Consistency of pituitary adenoma: Prediction by pharmacoki- netic dynamic contrast-enhanced MRI and comparison with histologic collagen content
}

\author{
Kiyohisa Kamimura 1,*, Masanori Nakajo ${ }^{1}$, Manisha Bohara 1, Daigo Nagano ${ }^{1}$, Yoshihiko Fukukura ${ }^{1}$, Shingo Fu- \\ jio $^{2}$, Tomoko Takajo ${ }^{2}$, Kazuhiro Tabata ${ }^{3}$, Takashi Iwanaga ${ }^{4}$, Hiroshi Imai ${ }^{5}$, Marcel Dominik Nickel ${ }^{6}$ and Ta- \\ kashi Yoshiura ${ }^{1}$
}

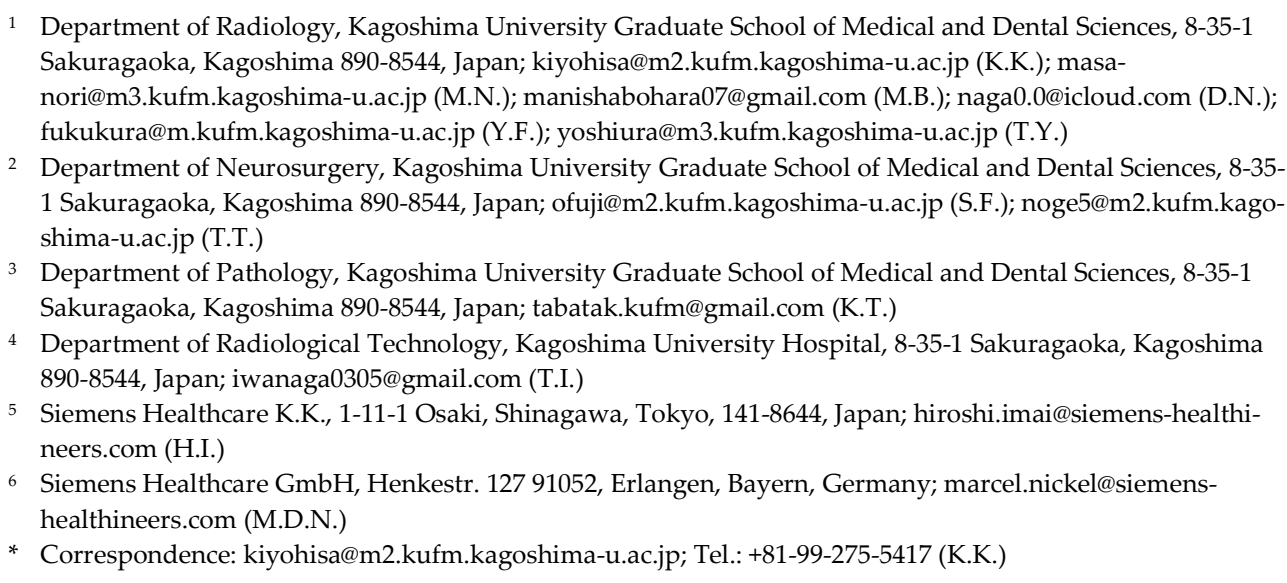

Simple Summary: Transsphenoidal resection of hard pituitary adenomas have a particularly high risk of residual tumor and complications. Therefore, prediction of tumor consistency is valuable for planning pituitary adenoma surgery. We prospectively examined whether quantitative pharmacokinetic analysis of dynamic contrast-enhanced magnetic resonance imaging (DCE-MRI) is useful for predicting consistency of pituitary adenoma in 49 patients. We found that the measure of volume of extravascular extracellular space per unit volume of tissue derived from DCE-MRI could predict the consistency of pituitary adenomas. Furthermore, the volume of extravascular extracellular space per unit volume of tissue was significantly positively correlated with histopathologic collagen content of the adenoma. Our results suggest that volume of extravascular extracellular space per unit volume of tissue derived from quantitative pharmacokinetic analysis of DCE-MRI has a predictive value for consistency of pituitary adenomas.

\begin{abstract}
Prediction of tumor consistency is valuable for planning transsphenoidal surgery for pituitary adenoma. A prospective study was conducted involving 49 participants with pituitary adenoma to determine whether quantitative pharmacokinetic analysis of dynamic contrast-enhanced magnetic resonance imaging (DCE-MRI) is useful for predicting consistency of adenoma. Pharmacokinetic parameters in the adenomas including volume of extravascular extracellular space (EES) per unit volume of tissue ( $\mathrm{Ve})$, blood plasma volume per unit volume of tissue $\left(\mathrm{V}_{\mathrm{p}}\right)$, volume transfer constant between blood plasma and EES ( $\left.\mathrm{K}^{\text {trans }}\right)$, and rate constant between EES and blood plasma $\left(\mathrm{k}_{\mathrm{ep}}\right)$ were obtained. The pharmacokinetic parameters and the histologic percentage of collagen content (PCC) were compared between soft and hard adenomas using Mann-Whitney $U$ test. Pearson's correlation coefficient was used to correlate pharmacokinetic parameters with PCC. Hard adenomas showed significantly higher PCC $(44.08 \pm 15.14 \%$ vs. $6.62 \pm 3.47 \%, p<0.01)$, ve $(0.332 \pm 0.124 \%$ vs. $0.221 \pm 0.104 \%, p=0.02)$, and $\mathrm{K}^{\text {trans }}(0.775 \pm 0.401 / \mathrm{min}$ vs. $0.601 \pm 0.612 / \mathrm{min}, p=0.02)$ than soft
\end{abstract}


adenomas. Moreover, a significant positive correlation was found between ve and PCC ( $\mathrm{r}=0.601, p$ $<0.01)$. The ve derived using DCE-MRI may have predictive value for consistency of pituitary adenoma.

Keywords: pituitary adenoma; consistency; magnetic resonance imaging; pharmacokinetic analysis; collagen. 


\section{Introduction}

Pituitary adenomas are the most common lesions of the sella turcica, comprising approximately $15 \%$ of all primary brain tumors [1]. Transsphenoidal surgery is among the standard initial treatments for pituitary adenoma [2]. The main limitation of this technique involves tumor consistency [3]. Most pituitary adenomas with soft consistency can be easily removed using this technique [4,5]. However, tumors with hard consistency may not be successfully debulked via the transsphenoidal approach [6]. Therefore, preoperative information regarding tumor consistency is helpful to neurosurgeons for planning an appropriate surgical approach that avoids or minimizes residual tumor and potential complications [5,6]. Consistency of pituitary adenomas has been considered to be relevant to collagen content [5-9].

The role of magnetic resonance imaging (MRI) in predicting consistency of pituitary adenoma is controversial. Several studies have suggested that relative signal intensity or signal intensity ratio of T1- or T2-weighted MRI and apparent diffusion coefficient (ADC) value correlate with tumor consistency, whereas others have concluded that they offer no predictive value [2,4-11].

Dynamic contrast-enhanced MRI (DCE-MRI) with pharmacokinetic analysis is a noninvasive and quantitative tool to assess the function of specific tissues [12]. The extended Tofts model is a commonly applied model for quantitative pharmacokinetic analysis [13-15], which generates four pharmacokinetic parameters: volume of extravascular extracellular space (EES) per unit volume of tissue (Ve), blood plasma volume per unit volume of tissue $\left(\mathrm{v}_{\mathrm{p}}\right)$, volume transfer constant between blood plasma and EES (Krans), and rate constant between EES and blood plasma (kep) [16]. DCE-MRI can be used to provide information concerning tumor microvascular distribution, perfusion, and permeability [17-19]. In addition, previous studies have reported that the fibrous content in pancreatic cancer is significantly correlated with the values of ve [18]. DCE-MRI of the pituitary gland is performed with a typical temporal resolution of $20 \mathrm{~s}$. Only a few attempts have been made to quantitatively analyze DCE-MRI data of the pituitary gland, which is presumably due to the limited temporal resolution [20]. Recent studies have demonstrated the feasibility of higher temporal resolution DCE-MRI using a prototype compressed sensing volumetric interpolated breath-hold examination (CS VIBE) sequence [21,22].

We hypothesized that ve measurement using the CS VIBE sequence would be useful in predicting the consistency of pituitary adenomas by identifying tumors with increased collagen fibrous content. This study aims to prospectively evaluate values of quantitative pharmacokinetic parameters to preoperatively predict the consistency of pituitary adenomas.

\section{Materials and Methods}

\subsection{Participant Characteristics}

The institutional review board approved this prospective cross-sectional study (approval no. 180255), and informed consent was obtained from each participant.

We included adult (age $>18$ years) women and men with pituitary lesions who underwent clinically indicated conventional and DCE-MRI between July 2018 and October 2020 at our institution. Exclusion criteria included pituitary lesions other than adenoma, and not undergoing tumorectomy. The hormonal activity of each pituitary adenoma was determined by preoperative measurement of serum hormone levels and pituitary provocation tests. 


\subsection{MRI Examinations}

All participants underwent MRI with a 3 T system (MAGNETOM Prisma; Siemens Healthcare, Erlangen, Germany), equipped with a 20-channel head/neck coil. Pre-contrast imaging of the sellar region included the following sequences (Table 1): coronal pre-contrast 2D T1-weighted spin-echo imaging, coronal 2D T2-weighted turbo spin-echo imaging, and coronal diffusion-weighted imaging using readout-segmented echo-planar, which reduces susceptibility and $\mathrm{T} 2 *$ blurring artifacts $[23,24]$.

For DCE-MRI, coronal T1-weighted imaging was performed using a prototype 3D CS VIBE sequence (Table 1). DCE-MRI was performed after intravenous administration of $0.1 \mathrm{mmol} / \mathrm{kg}$ meglumine gadoterate (Magnescope; Guerbet, Aulnay-sous-Bois, France) followed by $20 \mathrm{ml}$ of saline at a rate of $4 \mathrm{ml} / \mathrm{s}$.

Coronal post-contrast 3D T1-weighted spoiled gradient-echo imaging was performed immediately after the DCE-MRI scan.

Table 1. Pituitary MRI protocol.

\begin{tabular}{|c|c|c|c|c|c|}
\hline & Pre-contrast & T2-weighted & DWI & DCE imaging & Post-contrast \\
\hline & T1-weighted & imaging & & & T1-weighted \\
\hline & imaging & & & & imaging \\
\hline Orientation & Coronal & Coronal & Coronal & Coronal & Coronal \\
\hline Sequence & 2D SE & 2D TSE & RESOLVE & 3D VIBE & 3D FLASH \\
\hline $\mathrm{TR}(\mathrm{ms})$ & 450 & 4000 & 4000 & 3.9 & 4.68 \\
\hline $\mathrm{TE}(\mathrm{ms})$ & 12 & 95 & 49 & 0.97 & 1.74 \\
\hline FA (degree) & $70 / 180$ & $90 / 130$ & 150 & 10 & 11 \\
\hline Bandwidth (Hz/pixel) & 130 & 189 & 694 & 670 & 370 \\
\hline \multirow[t]{3}{*}{ Number of excitations } & 1 & 2 & 1 and 3 for & 1 & 2 \\
\hline & & & \multicolumn{3}{|l|}{ low and high } \\
\hline & & & $b$ values & & \\
\hline Turbo factor & N/A & 9 & N/A & N/A & $\mathrm{N} / \mathrm{A}$ \\
\hline Acceleration factor & 1 & 2 & 2 & N/A & N/A \\
\hline CS factor & $\mathrm{N} / \mathrm{A}$ & $\mathrm{N} / \mathrm{A}$ & $\mathrm{N} / \mathrm{A}$ & 7 & $\mathrm{~N} / \mathrm{A}$ \\
\hline
\end{tabular}




\begin{tabular}{|c|c|c|c|c|c|}
\hline $\mathrm{b}$ values $\left(\mathrm{s} / \mathrm{mm}^{2}\right)$ & N/A & N/A & 0 and 1000 & N/A & N/A \\
\hline Directions of motion- & N/A & N/A & 3 & N/A & N/A \\
\hline \multicolumn{6}{|l|}{ probing gradients } \\
\hline Readout segments & N/A & N/A & 9 & N/A & N/A \\
\hline $\mathrm{FOV}\left(\mathrm{mm}^{2}\right)$ & $180 \times 180$ & $180 \times 180$ & $120 \times 120$ & $159 \times 180$ & $200 \times 200$ \\
\hline Matrix & $240 \times 320$ & $313 \times 448$ & $84 \times 120$ & $163 \times 192$ & $320 \times 256$ \\
\hline Thickness (mm) & 3 & 3 & 2 & 0.9 & 0.6 \\
\hline Intersection gap (mm) & 0.3 & 0.3 & 0 & N/A & N/A \\
\hline Temporal resolution & N/A & N/A & N/A & 5 & N/A \\
\hline
\end{tabular}

(s)

$\begin{array}{lllll}\text { Acquisition time (s) } & 148 & 158 & 252 & 203\end{array}$

DWI: diffusion weighted image; DCE: dynamic contrast-enhanced; TR: repetition time; TE: echo time; FA: flip angle; CS: compressed sensing; FOV: field of view; SE: spin-echo; TSE: turbo spin-echo; RESOLVE: readout-segmented echo-planar; VIBE: volumetric interpolated breath-hold examination; FLASH: fast low-angle shot; N/A: not applicable.

\subsection{Measurement of Maximum Tumor Diameter and Volume}

A radiologist (D.N. with 3 years of radiological experience) manually measured the maximum tumor diameter on coronal post-contrast 3D T1-weighted images on a picture archiving and communication systems workstation (Synapse; Fujifilm Medical, Tokyo, Japan). The tumor volume was estimated using the equation developed by Di Chiro and Nelson (volume $=0.5 \times$ length $\times$ height $\times$ width) [25] .

\subsection{Pharmacokinetic Analysis of DCE-MRI}

All DCE-MRI images were processed using Vitrea (Canon Medical Systems Corporation, Otawara, Japan). The arterial input function was automatically detected at the internal carotid artery. The extended Tofts model was used to calculate tumor pharmacokinetic parameters of $\mathrm{Ve}_{\mathrm{e}} \mathrm{v}, \mathrm{K}^{\text {trans }}$, and $\mathrm{k}_{\mathrm{ep}}[16]$.

The pre-contrast T1-weighted MRIs, T2-weighted MRIs, ADC maps, and pharmacokinetic parameters were automatically co-registered to the post-contrast T1-weighted MRIs in Vitrea. Region of interests (ROIs) were first drawn on the contrast-enhanced MRIs by two independent radiologists (K.K. and M.B. with 23 and 5 years of radiological experience, respectively) who were blinded to participants' clinical information. These were then duplicated on the other image types. ROIs were manually annotated on the coronal slice with maximum lesion extent. Cystic, necrotic, and hemorrhagic areas were intentionally excluded. An additional round ROI (5 $\mathrm{mm}$ diameter) was placed in the normal- 
appearing white matter of the temporal lobe. The mean signal intensity or parametric value was obtained for each ROI.

For the conventional images, relative signal intensity of the pituitary adenoma was assessed by calculating the ratio of signal intensity on T1-weighted (rT1) and T2-weighted MRI (rT2) in the tumor to those in the normal-appearing white matter.

\subsection{Intraoperative Findings}

At surgery, tumor consistency was evaluated by a neurosurgeon (S.F. with 18 years of neurosurgery experience), who was blinded to the quantitative MRI findings. The tumors were classified into two groups: tumors with soft (easily removable through suction) and hard consistency (removable with difficulty through suction or not removable through suction but excisable piece by piece).

\subsection{Histologic Examination}

Histopathological assessments were performed by one pathologist (K.T. with 20 years of experience) who was blinded to the MRI data. Hematoxylin-eosin staining was performed routinely. Collagen content was evaluated using Azan staining. Percentage of collagen content (PCC) was obtained using the following methods. Azan-stained histopathological slides were scanned using Aperio CS2 (× 20 magnification; Leica Biosystems, Vista, CA), and images of five randomly chosen areas within the lesions were taken using $\times 400$ magnification. Each area of collagen and the total tumor area were measured, and PCC was calculated by using the following equation: PCC $=[\Sigma($ Acoll $) / \Sigma($ Atum $)] \times 100$, where Acoll is area of collagen and Atum is area of total tumor. Images of Azan-stained sections were analyzed using an image processing integration software (WinROOF2015, version 3.12.0; Mitani Corp., Tokyo, Japan). Azan-stained fields of $0.5 \times 0.5 \mathrm{~mm}^{2}$ were uploaded into the image analyzer, and each collagen content contour was automatically traced to measure PCC.

For functioning adenomas, we evaluated immunohistochemistry for diagnosis and classification of pituitary tumors according to the 2017 World Health Organization guidelines [1]. However, for clinically nonfunctioning adenoma, we did not evaluate immunohistochemistry.

\subsection{Statistical Analyses}

Statistical analyses were performed using MedCalc version 15.10.0 (MedCalc Software, Mariakerke, Belgium). The Mann-Whitney $U$ test and Fisher's exact test were used to compare participant characteristics. Interobserver agreement for MRI parameters was evaluated using intraclass correlation coefficients. Intraclass correlation coefficients of $>$ 0.74 were considered excellent agreement [26]. The values measured by the two observers were averaged for each ROI.

The MRI parameters and PCC of the hard and soft adenomas identified during surgery were compared using the Mann-Whitney $U$ test. Receiver operating characteristic curves were generated to calculate the area under the receiver operating characteristic curves, sensitivity, specificity, and accuracy. The maximum Youden index was used to determine the optimal cut-off points. The DeLong method was used to compare area under the receiver operating characteristic curve values [27].

Pearson's correlation coefficients were used to correlate PCC with the MRI parameters. $p$ values $<0.05$ indicated statistical significance.

\section{Results}

\subsection{Characteristics of Participants and Adenomas}


From 100 participants with pituitary lesions, the following participants were excluded: 26 who had pituitary lesions other than adenoma (histologically confirmed Rathke's cleft cyst $[n=11]$ and craniopharyngioma [ $n=6]$, meningioma $[n=4]$, germinoma $[n=2]$, and arachnoid cyst $[n=3]$ showing typical imaging findings), and 25 who had pituitary adenomas (nonfunctioning $[n=17]$ and lactotroph adenoma $[n=8]$ ) without undergoing tumorectomy. A total of 49 participants (mean age $55 \pm 17$ years; 23 men and 26 women) with pituitary adenoma were included in the final sample (Figure 1). Forty-eight participants were treated with the endoscopic endonasal transsphenoidal technique, and one participant underwent combined transsphenoidal and transcranial resection. Tumor consistency at surgery was classified as soft and hard in 34 (69.4\%) and 15 (30.6\%) participants, respectively.

Table 2 shows the characteristics of participants and adenomas. A total of 33 participants were diagnosed as nonfunctioning adenomas, 13 as somatotroph adenomas, 2 as thyrotroph adenomas, and 1 as a corticotroph adenoma. There were no significant differences in participant's age, gender, or hormonal function between soft and hard adenomas.

Representative cases are presented in Figures 2 and 3.

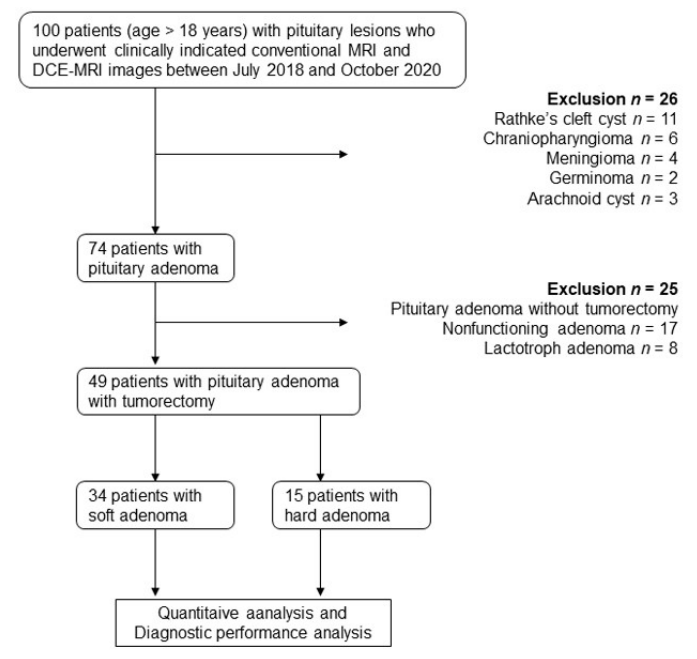

Figure 1. Study flowchart for the inclusion and exclusion criteria of the participant sample and pituitary lesion characterization.

DCE-MRI: dynamic contrast-enhanced MRI.

Table 2. Characteristics of participants and adenomas.

\begin{tabular}{lllll}
\hline Participants characteristics & Total & Soft Adenoma & Hard Adenoma & $p$ Value \\
& $(\mathbf{n}=49)$ & $(\mathbf{n}=\mathbf{3 4})$ & $\mathbf{( n = 1 5 )}$ & \\
& $55 \pm 17$ & $53 \pm 17$ & $60 \pm 17$ & $0.12^{*}$ \\
Age (y) & $23(46.9)$ & $15(44.1)$ & $8(53.3)$ & $0.76^{*}$ \\
No. of men & & & & \\
Maximum diameter $(\mathrm{mm})$ & $24.2 \pm 12.2$ & $24.8 \pm 14.0$ & $26.1 \pm 7.0$ & $0.27^{*}$
\end{tabular}




\begin{tabular}{|c|c|c|c|c|}
\hline Volume $\left(\mathrm{mm}^{3}\right)$ & $8090 \pm 13570$ & $8830 \pm 16070$ & $6420 \pm 4210$ & $0.18^{*}$ \\
\hline \multicolumn{5}{|l|}{ Pituitary lesions } \\
\hline Nonfunctioning & $33(67.3)$ & $21(63.6)$ & $12(36.4)$ & $0.32 \dagger$ \\
\hline Functioning & $16(32.7)$ & $13(81.3)$ & $3(18.7)$ & \\
\hline Somatotroph & 13 (26.5) & $10(77.0)$ & $3(23.0)$ & \\
\hline Thyrotroph & $2(4.1)$ & $2(100)$ & $0(0)$ & \\
\hline Corticotroph & $1(2.1)$ & $1(100)$ & $0(0)$ & \\
\hline
\end{tabular}

Statistical tests used: *Mann-Whitney $U$ test, + Fisher's exact test

A

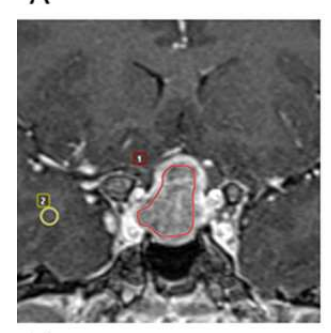

E

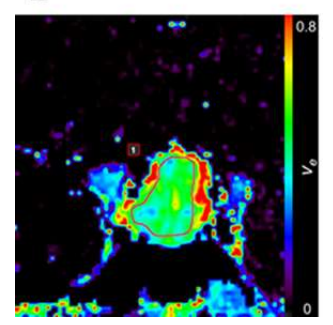

B

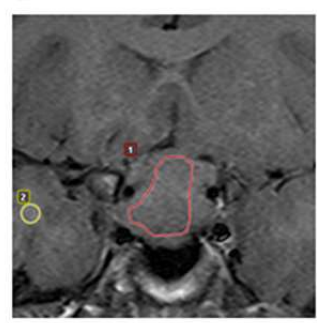

F

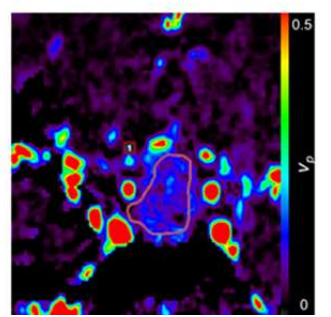

C

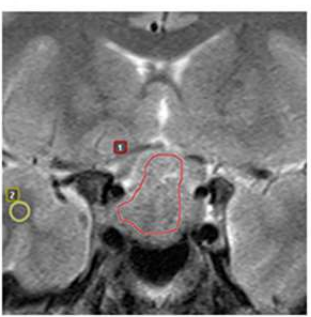

G

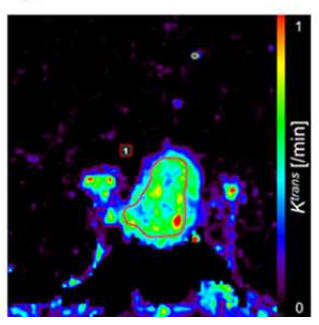

D

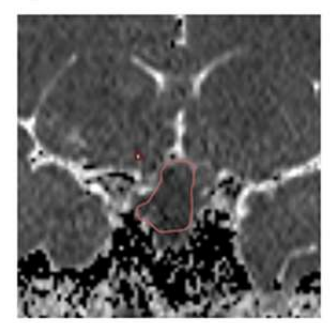

$\mathrm{H}$

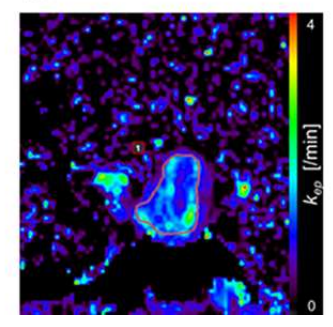

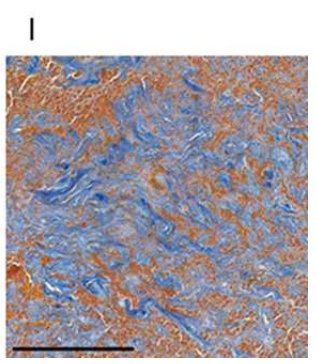

Figure 2. Pituitary adenomas with hard consistency. A contrast-enhanced coronal T1-weighted image of a pituitary adenoma extending into the suprasellar cistern (A). The adenoma is slightly hypointense in the white matter on the T1weighted image $(\mathrm{rT} 1=0.931 ; \mathbf{B})$ and hyperintense on the T2-weighted image $(\mathrm{rT} 2=1.71 ; \mathbf{C})$. The corresponding ADC map shows a low ADC value $\left(0.674 \times 10^{-3} \mathrm{~mm}^{2} / \mathrm{s} ; \mathrm{D}\right)$. Pharmacokinetic parametric mapping shows a high ve value $(0.440 ; \mathrm{E})$, a low $v_{p}$ value $(0.050 ; \mathbf{F})$, a high $\mathrm{K}^{\text {trans }}$ value $(0.615 / \mathrm{min} ; \mathrm{G})$, and a low $\mathrm{kep}_{\text {value }}(1.410 / \mathrm{min}$; $\mathbf{H})$ in the adenoma. ROIs for tumor and normal-appearing white matter are indicated by red and yellow lines, respectively. Specimen of tumor at pathologic examination shows conspicuous stromal fibrosis (in blue) within small cells (in red) (PCC $=50.9 \%$; I). Bar indicates $200 \mu \mathrm{m}$.

rT1: ratio of signal intensity on T1-weighted MRI; rT2: ratio of signal intensity on T2-weighted MRI; ADC: apparent diffusion coefficient; ve: volume of extravascular extracellular space per unit volume of tissue; vp: blood plasma volume per unit volume of tissue; $K^{\text {trans: }}$ volume transfer constant between blood plasma and extravascular extracellular space; kep: rate constant between extravascular extracellular space and blood plasma; ROI: region of interest; PCC: percentage of collagen content. 
A

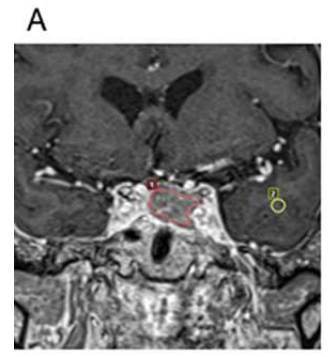

E

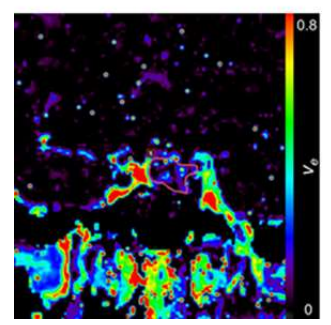

B

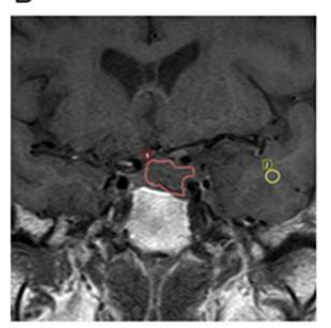

F

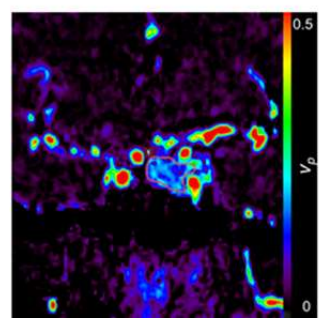

C

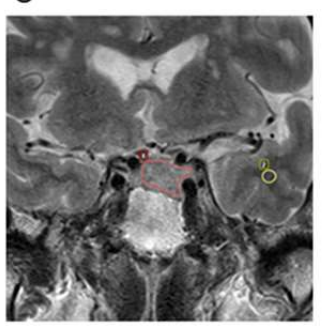

G

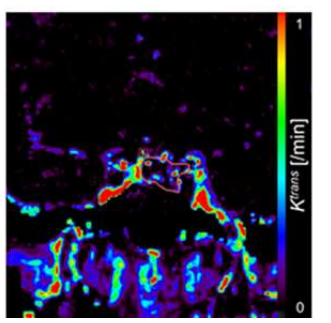

D

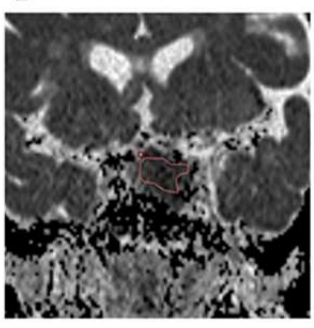

$\mathrm{H}$

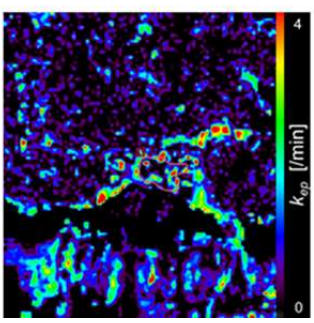

I

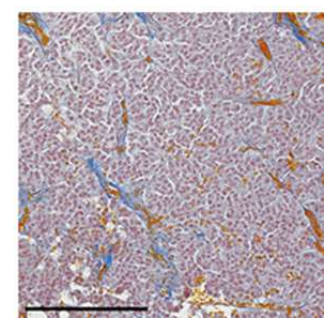

Figure 3. Pituitary adenoma with soft consistency. A contrast-enhanced coronal T1-weighted image of a pituitary adenoma extending into the left cavernous sinus (A). The adenoma is slightly hypointense in the white matter on the T1-weighted image (rT1 $=0.908 ; \mathbf{B})$ and hyperintense on the T2-weighted image (rT2 =1.901; C). The corresponding ADC map shows a low ADC value $\left(0.854 \times 10^{-3} \mathrm{~mm}^{2} / \mathrm{s} ; \mathrm{D}\right)$. Parametric mapping shows a low ve value $(0.105 ; \mathrm{E})$, a high $\mathrm{vp}$ value $(0.145 ; \mathbf{F})$, a low $\mathrm{K}^{\text {trans }}$ value $(0.175 / \mathrm{min} ; \mathrm{G})$, and a low $\mathrm{k}_{\mathrm{ep}}$ value $(1.245 / \mathrm{min} ; \mathrm{H})$ in the adenoma. ROIs for tumor and normal-appearing white matter are indicated by red and yellow lines, respectively. Pathologic examination shows scant fibrous stroma (in blue) within small cells (in red) (PCC $=7.4 \%$; I). Bar indicates $200 \mu \mathrm{m}$.

rT1: ratio of signal intensity on T1-weighted MRI; rT2: ratio of signal intensity on T2-weighted MRI; ADC: apparent diffusion coefficient; ve: volume of extravascular extracellular space per unit volume of tissue; vp: blood plasma volume per

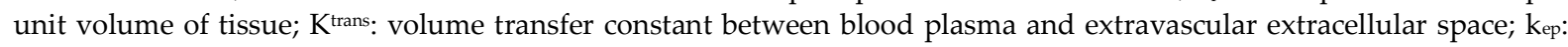
rate constant between extravascular extracellular space and blood plasma; ROI: region of interest; PCC: percentage of collagen content.

\subsection{Maximum Diameter and Volume of Pituitary Adenomas}

The maximum diameter of pituitary adenomas ranged from 6 to $69 \mathrm{~mm}$. Three adenomas $(6 \%)$ were smaller than $10 \mathrm{~mm}$ in maximum diameter, while the remaining 46 $(94 \%)$ were larger than $10 \mathrm{~mm}$. There was no significant difference in maximum diameter between soft and hard adenomas $(24.8 \pm 14.0 \mathrm{~mm}$ vs. $26.1 \pm 7.0 \mathrm{~mm}, p=0.27$; Table 2$)$. The volume of pituitary adenomas ranged from 87.5 to $68930 \mathrm{~mm}^{3}$. There was no significant difference in volume between soft and hard adenomas $\left(8830 \pm 16070 \mathrm{~mm}^{3}\right.$ vs. $6420 \pm 4210$ $\mathrm{mm}^{3}, p=0.18$; Table 2).

\subsection{Interobserver Agreement}

The intraclass correlation coefficients and $95 \%$ confidence intervals for rT1, rT2, ADC, $\mathrm{V}_{\mathrm{e},} \mathrm{V}_{\mathrm{p}}, \mathrm{K}^{\text {trans }}$, and $\mathrm{k}_{\text {ep }}$ were 0.778 ( 0.638 to 0.868$), 0.940$ (0.895 to 0.965$), 0.818$ (0.699 to 0.893 ), 0.923 ( 0.867 to 0.956$), 0.980$ ( 0.965 to 0.989$), 0.917$ ( 0.857 to 0.952$)$, and 0.882 ( 0.800 to 0.932 ), respectively, which indicated excellent agreement for all measures.

\subsection{Comparisons of Imaging and Histologic Parameters between Soft and Hard Adenomas}

Mean values of the MRI parameters and PCC of the histological examination for soft and hard adenomas are shown in Table 3.

Hard adenomas had significantly higher PCC than soft adenomas $(44.08 \pm 15.14$ vs. $6.62 \pm 3.47, p<0.01)$. Hard adenomas had significantly higher ve $(0.332 \pm 0.124$ vs. $0.221 \pm$ 
$0.104, p=0.02)$ and $\mathrm{K}^{\text {trans }}(0.775 \pm 0.401 / \mathrm{min}$ vs. $0.601 \pm 0.612 / \mathrm{min}, p=0.02)$ values than soft adenoma, whereas there were no significant differences in rT1 (0.854 \pm 0.086 vs. $0.840 \pm$ $0.077, p=0.40), \mathrm{rT} 2(1.580 \pm 0.295$ vs. $1.661 \pm 0.453, p=0.55), \operatorname{ADC}\left(0.769 \pm 0.201 \times 10^{-3} \mathrm{~mm}^{2} / \mathrm{s}\right.$ vs. $\left.0.832 \pm 0.263 \times 10^{-3} \mathrm{~mm}^{2} / \mathrm{s}, p=0.47\right)$, $\mathrm{vp}_{\mathrm{p}}(0.040 \pm 0.042$ vs. $0.070 \pm 0.084, p=0.36)$, or $\mathrm{k}_{\mathrm{ep}}$ $(2.641 \pm 1.672 / \mathrm{min}$ vs. $2.240 \pm 1.691 / \mathrm{min}, p=0.37)$ values (Table 3$)$.

Table 3. MRI parameters and histologic percentage of collagen content of pituitary adenomas.

\begin{tabular}{|c|c|c|c|}
\hline & \multicolumn{2}{|c|}{ Tumor consistency group } & \multirow[b]{2}{*}{$p$ value } \\
\hline & Soft adenoma $(n=34)$ & Hard adenoma $(n=15)$ & \\
\hline rT1 & $0.840 \pm 0.077$ & $0.854 \pm 0.086$ & $0.40^{*}$ \\
\hline rT2 & $1.661 \pm 0.453$ & $1.580 \pm 0.295$ & $0.55^{*}$ \\
\hline $\operatorname{ADC}\left(10^{-3} \mathrm{~mm}^{2} / \mathrm{s}\right)$ & $0.832 \pm 0.263$ & $0.769 \pm 0.201$ & $0.47^{*}$ \\
\hline Ve & $0.221 \pm 0.104$ & $0.332 \pm 0.124$ & $0.02^{*}$ \\
\hline $\mathrm{V}_{\mathrm{p}}$ & $0.070 \pm 0.084$ & $0.040 \pm 0.042$ & $0.36^{*}$ \\
\hline $\mathrm{K}^{\text {trans }}(/ \min )$ & $0.601 \pm 0.612$ & $0.775 \pm 0.401$ & $0.02^{*}$ \\
\hline $\mathrm{k}_{\mathrm{ep}}(/ \mathrm{min})$ & $2.240 \pm 1.691$ & $2.641 \pm 1.672$ & $0.37^{*}$ \\
\hline PCC (\%) & $6.62 \pm 3.47$ & $44.08 \pm 15.14$ & $<0.01^{*}$ \\
\hline
\end{tabular}

rT1: ratio of signal intensity on T1-weighted MRI; rT2: ratio of signal intensity on T2-weighted MRI; ADC: apparent diffusion coefficient; ve: volume of extravascular extracellular space per unit volume of tissue; vp: blood plasma volume per unit volume of tissue; Ktrans: volume transfer constant between blood plasma and extravascular extracellular space; kep: rate constant between extravascular extracellular space and blood plasma; PCC: percentage of collagen content.

Statistical tests used: *Mann-Whitney $U$ test.

\subsection{MRI Parameters Correlated with Percentage of Collagen Content in Pituitary Adenomas}

We found a significant positive correlation between ve and PCC in pituitary adenomas $(n=49, \mathrm{r}=0.601, p<0.01$; Figure 4). No other MRI parameters showed a significant correlation with PCC ( $\mathrm{rT} 1[\mathrm{r}=0.035, p=0.81], \mathrm{rT2}[\mathrm{r}=-0.075, p=0.61]$, ADC [r $=0.044, p=$ $0.76], \mathrm{v}_{\mathrm{p}}[\mathrm{r}=-0.165, p=0.26], \mathrm{K}^{\text {trans }}[\mathrm{r}=0.057, p=0.70]$, and $\left.\mathrm{kep}_{\mathrm{p}}[\mathrm{r}=-0.055, p=0.71]\right)$. 


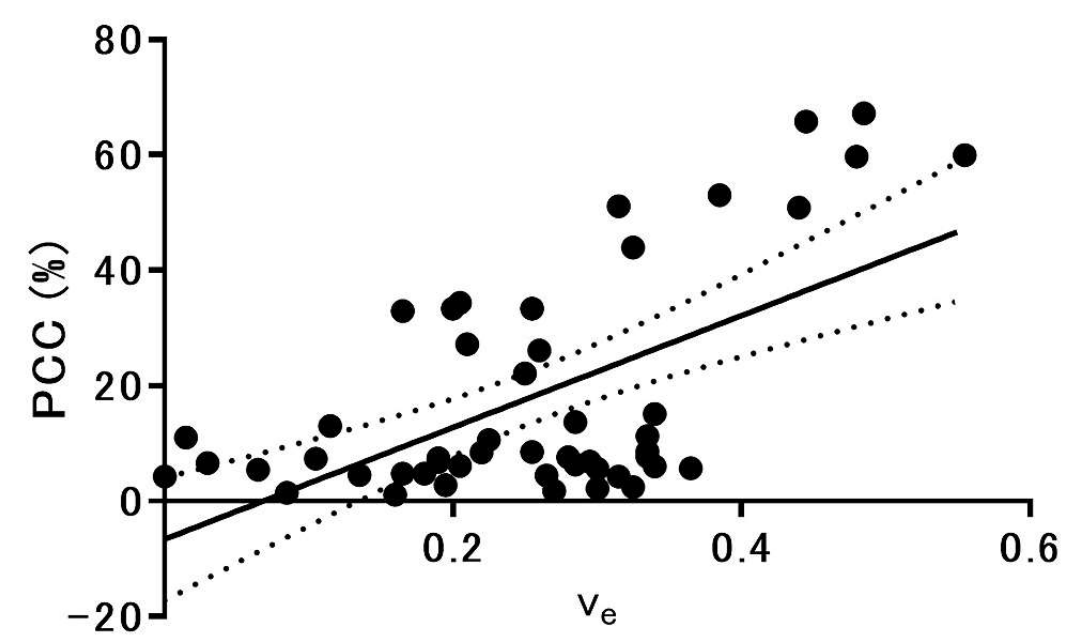

Figure 4. Scatterplot shows the relationship between ve and the PCC in 49 pituitary adenomas. The solid and dotted lines indicate the linear regression line and $95 \%$ confidence intervals.

Ve: volume of extravascular extracellular space per unit volume of tissue; PCC: percentage of collagen content.

\subsection{Diagnostic Performance}

Figure 5 shows the receiver operating characteristic curves for the MRI parameters. The area under the receiver operating characteristic curves for rT1, rT2, ADC, ve, $\mathrm{v}_{\mathrm{p}}, \mathrm{K}^{\text {trans }}$, and $k_{\text {ep }}$ were $0.578,0.555,0.566,0.712,0.583,0.703$, and 0.582 , respectively (Table 4). Among the MRI parameters, ve was the most discriminative quantitative measurement for predicting hard adenomas; however, the area under the receiver operating characteristic curve for ve did not significantly differ from other parameters. Table 4 shows sensitivity, specificity, and accuracy at the optimal cut-off values of each MRI parameter for the diagnosis of hard adenomas. Among all MRI parameters, ve showed the highest accuracy $(81.6 \%)$ among all MRI parameters.

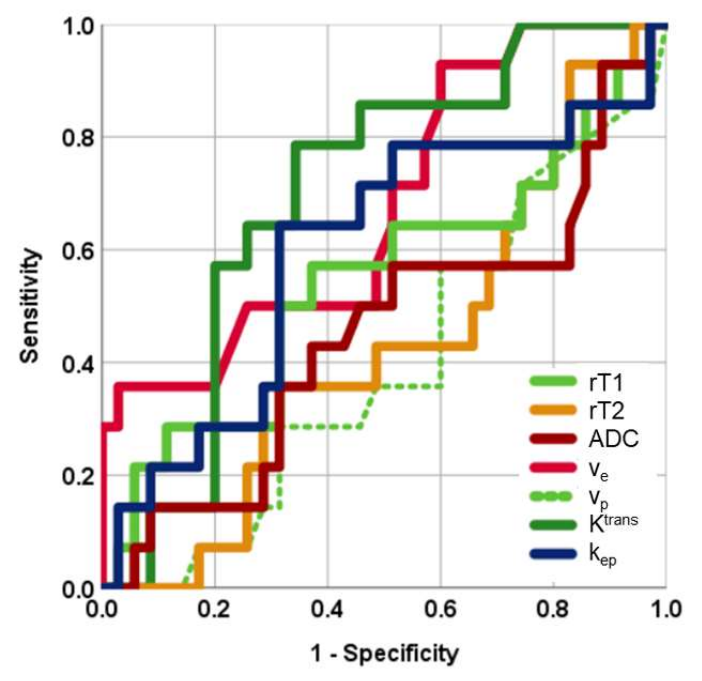

Figure 5. Receiver operating characteristic curve of each parameter for differentiating hard adenomas from soft adenomas (34 soft adenomas and 15 hard adenomas). 
rT1: ratio of signal intensity on T1-weighted MRI; rT2: ratio of signal intensity on T2-weighted MRI; ADC: apparent diffusion coefficient; ve: volume of extravascular extracellular space per unit volume of tissue; $\mathrm{v}_{\mathrm{p}}$ : blood plasma volume per unit volume of tissue; $K^{\text {trans: }}$ volume transfer constant between blood plasma and extravascular extracellular space; kep: rate constant between extravascular extracellular space and blood plasma.

Table 4. Diagnostic performance of MRI parameters in detecting hard adenomas.

\begin{tabular}{|c|c|c|c|c|c|}
\hline & AUC & Optimal cut-off value & Sensitivity (\%) & Specificity (\%) & Accuracy (\%) \\
\hline rT1 & 0.578 & 0.866 & $60.0(9 / 15)$ & $64.7(22 / 34)$ & $63.3(31 / 49)$ \\
\hline rT2 & 0.555 & 1.551 & $53.3(8 / 15)$ & $64.7(22 / 34)$ & $61.2(30 / 49)$ \\
\hline $\mathrm{ADC}$ & 0.566 & $0.674\left(10^{-3} \mathrm{~mm}^{2} / \mathrm{s}\right)$ & $46.7(7 / 15)$ & $73.5(25 / 34)$ & $65.3(32 / 49)$ \\
\hline $\mathrm{Ve}$ & 0.712 & 0.365 & $40.0(6 / 15)$ & $100(34 / 34)$ & $81.6(40 / 49)$ \\
\hline $\mathrm{v}_{\mathrm{p}}$ & 0.583 & 0.100 & $93.3(14 / 15)$ & $26.5(9 / 34)$ & $46.9(23 / 49)$ \\
\hline $\mathrm{K}^{\text {trans }}$ & 0.703 & $0.560(/ \mathrm{min})$ & $80.0(12 / 15)$ & $67.7(23 / 34)$ & $71.4(35 / 49)$ \\
\hline kep & 0.582 & $0.277(/ \mathrm{min})$ & $60.0(9 / 15)$ & $67.7(23 / 34)$ & $65.3(40 / 49)$ \\
\hline
\end{tabular}

rT1: ratio of signal intensity on T1-weighted MRI; rT2: ratio of signal intensity on T2-weighted MRI; ve: volume of extravascular

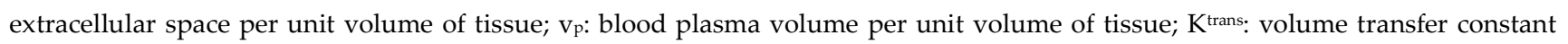
between blood plasma and extravascular extracellular space; kep: rate constant between extravascular extracellular space and blood plasma.

\section{Discussion}

The present study suggests that preoperative mapping of volume of extravascular extracellular space per unit volume of tissue derived from pharmacokinetic dynamic contrast-enhanced MRI is a useful imaging method for predicting the consistency of pituitary adenomas. Tumor consistency can critically affect surgical resectability of pituitary adenomas in participants who have been treated using a transsphenoidal endoscopic technique $[5,6]$. In most cases, pituitary adenoma has a soft consistency, and thus, aspiration and curettage are typically used to remove the tumor. However, $5 \%-15 \%$ of large pituitary adenomas have a hard consistency [9], and these tumors require resection using a surgical knife [28], which may increase the risk of complications, such as damage of the healthy pituitary gland, internal carotid artery, and optic nerve. Moreover, transsphenoidal resection of hard adenomas can result in residual tumor tissue and may require a second-look surgery, radiotherapy, or additional transcranial surgery. Therefore, imaging prediction of tumor consistency is valuable for planning transsphenoidal surgery for pituitary adenoma. To the best of our knowledge, this is the first study to reveal the utility of 
quantitative pharmacokinetic analysis of dynamic contrast-enhanced MRI in predicting the consistency of pituitary adenoma.

Consistency of pituitary adenoma has been considered to be relevant to collagen content $[5,8,9,28]$. This study confirmed a relative abundancy of collagen, as measured by histopathological PCC, in hard adenomas compared with soft adenomas. In pancreatic cancer, extracellular fibrosis content has been shown to be positively correlated to ve obtained using pharmacokinetic analysis of DCE-MRI [18]. Collagen content is also one of the extravascular extracellular matrices in pituitary adenomas; thus, it is conceivable that higher PCC is associated with an increase in ve. As expected, this study revealed that ve was positively correlated with PCC in pituitary adenomas.

Despite the routine implementation of DCE-MRI as a preoperative MRI protocol for pituitary adenoma, quantitative pharmacokinetic analysis of pituitary DCE-MRI has rarely been reported. Zhai et al [20] and Liu et al [29] performed pharmacokinetic analysis of pituitary DCE-MRI using T1-weighted gradient-echo pulse sequences with temporal resolutions of 8 to $8.5 \mathrm{~s}$ and an in-plane resolution of $<1 \mathrm{~mm}^{2}$ but comparatively thicker slices ranging from 2.5 to $3 \mathrm{~mm}$. In this study, we employed the compressed sensing incorporated in a 3D gradient-echo sequence to enable both higher temporal resolution (5 s) and submillimeter isotropic voxels $\left(0.9 \times 0.9 \times 0.9 \mathrm{~mm}^{3}\right)$ [30]. This type of imaging technique allows quantitative pharmacokinetic DCE-MRI of smaller pituitary lesions.

In addition to the pharmacokinetic parameters, we tested the possible utility of conventional MRI parameters for the prediction of pituitary adenoma consistency. Previous studies have reported conflicting results. Ma et al [8] showed that relative signal intensity (tumor to frontal lobe white matter) on pre-contrast T1-weighted images are useful in predicting the consistency of pituitary adenoma, whereas other studies have suggested that there is no relationship between tumor consistency and signal intensity on T1-weighted MRIs [2-6]. Furthermore, another study described an inverse correlation between signal intensity on T2-weighted MRIs and collagen content, which was abundant in hard pituitary adenomas [9]. However, several studies have failed to show such a relationship [27]. One study demonstrated that lower ADC values are correlated with softer tumor consistency at surgery and with higher cellularity at pathology [5]. In contrast, there has also been a report that showed that lower ADC values are correlated with harder tumor consistency and higher collagen content [7]. We did not find significant correlations between rT1, rT2, and ADC maps with tumor consistency or collagen content.

This study has some limitations. First is the use of a small sample size. Further studies should study a larger sample of participants to validate the potential threshold value of Ve for tumor resectability using aspiration. Second is the subjective classification of tumor consistency. Assessments were carried out by a single operating surgeon, so possible observer bias cannot be excluded. However, the relationship between ve and tumor consistency was supported by the significant positive correlation between ve and histological collagen content.

\section{Conclusions}

We performed quantitative pharmacokinetic analysis of pituitary adenomas using compressed sensing-based high-temporal resolution dynamic contrast-enhanced MRI. Our results suggest that volume of extravascular extracellular space per unit volume of tissue derived from quantitative pharmacokinetic analysis provide information about the consistency of pituitary adenomas.

Author Contributions: Conceptualization, K.K. and T.Y.; methodology, K.K. and Y.F.; software, H.I. and M.D.N.; validation, K.K., M.B. and D.N.; formal analysis, K.K.; investigation, K.K.; resources, K.K.; data curation, K.K., M.N., D.N., S.F., T.T., K.T., and T.I.; writing-original draft preparation, K.K.; writing - review and editing, T.Y.; visualization, K.K. and T.T.; supervision, K.K.; project administration, K.K. All authors have read and agreed to the published version of the manuscript.

Funding: This research received no external funding 
Institutional Review Board Statement: The study was conducted according to the guidelines of the Declaration of Helsinki, and approved by the Ethics Committee of Kagoshima University Hospital (approval number 180255, from 1 July 2018)

Informed Consent Statement: Informed consent was obtained from all subjects involved in the study.

Data Availability Statement: The data presented in this study are available on request from the corresponding authors. The data are not publicly available due to restrictions of the institutional Ethics Committee statement.

Acknowledgments: The authors wish to thank the staffs of Kagoshima University Hospital for their support.

Conflicts of Interest: The authors declare no conflict of interest.

\section{References}

1. Osamura R. Y.; Lopes M.B.S.; Grossman A.; Kontogeorgos G.; Trouillas J. Tumors of the pituitary gland. In WHO Classification of Tumors of Endocrine Organs, 4th ed.; Ricardo V.L.; Osamura R.Y.; Gunter K.; Juan R., Eds.; Lyon: International Agency for Research on Cancer: Lyon, France, 2017; pp. 11-64.

2. Boxerman J.L.; Rogg J.M.; Donahue J.E.; Machan J.T.; Goldman M.A.; Doberstein C.E. Preoperative MRI evaluation of pituitary macroadenoma: imaging features predictive of successful transsphenoidal surgery. AJR Am J Roentgenol. 2010, 195, 720-728, doi:10.2214/AJR.09.4128.

3. Snow R.B.; Lavyne M.H.; Lee B.C.; Morgello S.; Patterson R.H. Jr. Craniotomy versus transsphenoidal excision of large pituitary tumors: the usefulness of magnetic resonance imaging in guiding the operative approach. Neurosurgery. 1986, 19, 59-64, doi:10.1227/00006123-198607000-00008.

4. Alimohamadi M.; Sanjari R.; Mortazavi A.; Shirani M.; Tabriz M.H.; Kharazi H.H.; Amirjamshidi A. Predictive value of diffusion-weighted MRI for tumor consistency and resection rate of nonfunctional pituitary macroadenomas. Acta Neurochir. 2014, 156, 2245-2252, doi:10.1007/s00701-014-2259-6.

5. Pierallini A.; Caramia F.; Falcone C.; Tinelli E.; Paonessa A.; Ciddio B.A.; Fiorelli M.; Bianco F.; Natalizi S.; Ferrante L.; et al. Pituitary macroadenomas: preoperative evaluation of consistency with diffusion-weighted MR imaging-initial experience. Radiology. 2006, 239, 223-231, doi:10.1148/radiol.2383042204.

6. Romano A.; Coppola V.; Lombardi M.; Lavorato L.; Di Stefano D.; Caroli E.; Rossi Espagnet MC.; Tavanti F.; Minniti G.; Trillò G.; et al. Predictive role of dynamic contrast enhanced T1-weighted MR sequences in pre-surgical evaluation of macroadenomas consistency. Pituitary. 2017, 20, 201-209, doi:10.1007/s11102-016-0760-z.

7. Yiping L.; Ji X.; Daoying G.; Bo Y. Prediction of the consistency of pituitary adenoma: A comparative study on diffusionweighted imaging and pathological results. J Neuroradiol. 2016, 43, 186-194, doi:10.1016/j.neurad.2015.09.003.

8. Ma Z.; He W.; Zhao Y.; Yuan J.; Zhang O.; Wu Y.; Chen H.; Yao Z.; Li S.; Wang Y. Predictive value of PWI for blood supply and T1-spin echo MRI for consistency of pituitary adenoma. Neuroradiology. 2016, 58, 51-57, doi:10.1007/s00234-015-1591-8.

9. Iuchi T.; Saeki N.; Tanaka M.; Sunami K.; Yamaura A. MRI prediction of fibrous pituitary adenomas. Acta Neurochir. 1998, 140, 779-786, doi:10.1007/s007010050179.

10. Suzuki C.; Maeda M.; Hori K.; Kozuka Y.; Sakuma H.; Taki W.; Takeda K. Apparent diffusion coefficient of pituitary macroadenoma evaluated with line-scan diffusion-weighted imaging. J Neuroradiol. 2007, 34, 228-235, doi:10.1016/j.neurad.2007.06.007.

11. Mahmoud O.M.; Tominaga A.; Amatya V.J.; Ohtaki M.; Sugiyama K.; Saito T.; Sakoguchi T.; Kinoshita Y.; Shrestha P.; Abe N.; et al. Role of PROPELLER diffusion-weighted imaging and apparent diffusion coefficient in the evaluation of pituitary adenomas. Eur J Radiol. 2011, 80, 412-417, doi:10.1016/j.ejrad.2010.05.023.

12. Choyke P.L.; Dwyer A.J.: Knopp M.V. Functional tumor imaging with dynamic contrast-enhanced magnetic resonance imaging. J Magn Reson Imaging. 2003, 17, 509-520, doi:10.1002/jmri.10304.

13. Sourbron S.P.; Buckley D.L. On the scope and interpretation of the Tofts models for DCE-MRI. Magn Reson Med. 2011, 66, 735745, doi:10.1002/mrm.22861.

14. Zhang N.; Zhang L.; Qiu B.; Meng L.; Wang X.; Hou B.L. Correlation of volume transfer coefficient Ktrans with histopathologic grades of gliomas. J Magn Reson Imaging. 2012, 36, 355-363, doi:10.1002/jmri.23675.

15. Lu S.; Gao Q.; Yu J.; Li Y.; Cao P.; Shi H.; Hong X. Utility of dynamic contrast-enhanced magnetic resonance imaging for differentiating glioblastoma, primary central nervous system lymphoma and brain metastatic tumor. Eur J Radiol. 2016, 85, 17221727, doi:10.1016/j.ejrad.2016.07.005.

16. Tofts P.S.; Brix G.; Buckley D.L.; Evelhoch L.J.; Henderson E.; Knopp V.M.; Larsson B.H.; Lee Y.T.; Mayr A.N.; Parker J.G.; et al. Estimating kinetic parameters from dynamic contrast-enhanced T(1)-weighted MRI of a diffusable tracer: standardized quantities and symbols. J Magn Reson Imaging. 1999, 10, 223-232, doi:10.1002/(SICI)1522-2586(199909)10:3<223::AID-JMRI2>3.0.CO;2S.

17. Akisik M.F.; Sandrasegaran K.; Bu G.; Lin C.; Hutchins G.D.; Chiorean E.G. Pancreatic cancer: utility of dynamic contrast-enhanced MR imaging in assessment of antiangiogenic therapy. Radiology. 2010, 256, 441-449, doi:10.1148/radiol.10091733. 
18. Ma W.; Li N.; Zhao W.; Ren J.; Wei M.; Yang Y.; Wang Y.; Fu X.; Zhang Z.; Larson C.A.; et al. Apparent diffusion coefficient and dynamic contrast-enhanced magnetic resonance imaging in pancreatic cancer: characteristics and correlation with histopathologic parameters. J Comput Assist Tomogr. 2016, 40, 709-716, doi:10.1097/RCT.0000000000000434.

19. Anzalone N.; Castellano A.; Cadioli M.; Conte G.M.; Cuccarini V.; Bizzi A.; Grimaldi M.; Costa A.; Grillea G.; Vitali P.; et al. Brain gliomas: multicenter standardized assessment of dynamic contrast-enhanced and dynamic susceptibility contrast MR images. Radiology. 2018, 287, 933-943, doi:10.1148/radiol.2017170362.

20. Zhai J.; Zheng W.; Zhang Q.; Wu J.; Zhang X. Pharmacokinetic analysis for the differentiation of pituitary microadenoma subtypes through dynamic contrast-enhanced magnetic resonance imaging. Oncol Lett. 2019, 17, 4237-4244, doi:10.3892/ol.2019.10083.

21. Kamimura K.; Nakajo M.; Yoneyama T.; Bohara M.; Nakanosono R.; Fujio S.; Iwanaga T.; Nickel D.M.; Imai H.; Fukukura Y.; et al. Quantitative pharmacokinetic analysis of high-temporal-resolution dynamic contrast-enhanced MRI to differentiate the normal-appearing pituitary gland from pituitary macroadenoma. Jpn J Radiol. 2020, 38, 649-657, doi:10.1007/s11604-020-00942-4.

22. Honda M.; Kataoka M.; Onishi N.; Iima M.; Ohashi A.; Kanao S.; Nickel D.M.; Toi M.; Togashi K. New parameters of ultrafast dynamic contrast-enhanced breast MRI using compressed sensing. J Magn Reson Imaging. 2020, 51, 164-174, doi:10.1002/jmri.26838.

23. Koyasu S.; Iima M.; Umeoka S.; Morisawa N.; Porter A.D.; Ito J.; Bihan L.D.; Togashi K. The clinical utility of reduced-distortion readout-segmented echo-planar imaging in the head and neck region: initial experience. Eur Radiol. 2014, 24, 3088-3096, doi:10.1007/s00330-014-3369-5.

24. Holdsworth S.J.; Skare S.; Newbould R.D.; Guzmann R.; Blevins N.H.; Bammer R. Readout-segmented EPI for rapid high resolution diffusion imaging at 3T. Eur J Radiol. 2008, 65, 36-46, doi:10.1016/j.ejrad.2007.09.016.

25. Di Chiro G.; Nelson K.B. The volume of the sella turcica. Am J Roentgenol Radium Ther Nucl Med. 1962, 87, 989-1008.

26. Shrout P.E.; Fleiss J.L. Intraclass correlations: uses in assessing rater reliability. Psychol Bull. 1979, 86, 420-428, doi:10.1037/00332909.86.2.420.

27. DeLong E.R.; DeLong D.M.; Clarke-Pearson D.L. Comparing the areas under two or more correlated receiver operating characteristic curves: a nonparametric approach. Biometrics. 1988, 44, 837-845.

28. Yamamoto J.; Kakeda S.; Shimajiri S.; Takahashi M.; Watanabe K.; Kai Y.; Moriya J.; Korogi Y.; Nishizawa S. Tumor consistency of pituitary macroadenomas: predictive analysis on the basis of imaging features with contrast-enhanced 3D Fiesta at 3T. AJNR Am J Neuroradiol. 2014, 35, 297-303, doi:10.3174/ajnr.A3667.

29. Liu Y.Q.; Gao B.B.; Dong B.; Cheriyath P.S.S.; Song W.Q.; Xu B.; Wei Q.; Xie Z.L.; Guo Y.; Miao W.Y. Preoperative vascular heterogeneity and aggressiveness assessment of pituitary macroadenoma based on dynamic contrast-enhanced MRI texture analysis. Eur J Radiol. 2020, 129, 109125, doi:10.1016/j.ejrad.2020.109125.

30. Vreemann S.; Rodriguez-Ruiz A.; Nickel D.; Heacock L.; Appelman L.; van Zelst J.; Karssemeijer N.; Weiland E.; Maas M.; Moy L.; et al. Compressed sensing for breast MRI: resolving the trade-off between spatial and temporal resolution. Invest Radiol. 2017, 52:574-582, doi:10.1097/RLI.0000000000000384. 